\title{
El Aprendizaje Significativo y la Investigación en los Programas de Postgrado en Bolivia
}

\author{
Niver Montes Camacho \\ nmontesc57@gmail.com \\ Msc. Auditor financiero \\ Estudiante de Doctorado \\ Universidad Nacional Siglo XX, Bolivia
}

\begin{abstract}
RESUMEN
En Bolivia, como en los países de América Latina, se han creado muchos programas de postgrado, donde la investigación científica está ausente en gran parte debido a deficiencias del aprendizaje. Esto lleva a plantear problemas en la educación de postgrado que no estimula, e incluso desmotiva la investigación y la producción científica. La falta de cultura investigativa inhibe la investigación y crea híbridos que no son ni buenos profesionales ni buenos investigadores. Una de las respuestas a este problema es el aprendizaje significativo que se entiende como un aprendizaje con sentido.
\end{abstract}

Palabras clave: Bolivia, postgrado, Aprendizaje significativo, investigación científica 


\title{
Meaningful Learning and research in Postgraduate Programs in Bolivia
}

\begin{abstract}
In Bolivia, as in Latin American countries, many postgraduate programs have been created, where scientific research is mostly absent due to learning disabilities. This leads to propose problems in postgraduate education which do not stimulate, and even discourage research and scientific production. The lack of research culture inhibits research and creates hybrids who are neither good professionals nor good researchers. One of the answers to this problem is meaningful learning, which is understood as a meaningful learning.
\end{abstract}

Keywords: Bolivia, postgraduate, meaningful learning, scientific research

Artículo recibido: 27 marzo 2021 Aceptado para publicación: 30 abril 2021 Correspondencia: nmontesc57@gmail.com Conflictos de Interés: Ninguna que declarar 


\section{INTRODUCCION}

La formación y el acrecentamiento del conocimiento humano constituyen las nobles misiones de la universidad. La sociedad espera que la universidad no solamente forme personas con valores humanistas y competencias profesionales, sino también que en ella se cultive, preserve y acreciente el acervo del conocimiento humano a través de la investigación científica y tecnológica.

Una universidad que no investiga y no explora el campo de la ciencia y tecnología, no cumple con los requerimientos que le son demandados en el mundo moderno. La universidad se constituye como una entidad que por antonomasia es eminentemente productora (y divulgadora) de la ciencia, tecnología e innovación, mediante la realización de actividades correspondientes a la investigación científica. Las universidades mejor posicionadas del mundo son aquellas que realizan en dimensión considerable la actividad científica y tecnológica.

El postgrado se ha vuelto en el actual escenario internacional una prioridad para los gobiernos y los sectores productivos nacionales en términos de su contribución a la competitividad internacional y al desarrollo nacional. Tanto los gobiernos como los sectores de la producción demandan a las universidades una formación de recursos humanos altamente calificados, así como también de una producción científica y tecnológica de calidad que motiva un escenario de debate y reconfiguración de las políticas de postgrado.

Los estudios de postgrado constituyen una etapa final de la formación profesional, la cual puede darse con diferentes objetivos, modos y metas: la especialización, la maestría, el doctorado y, de manera más reciente, el postdoctorado. Cada uno de estos programas tiene diferentes finalidades.

En la actualidad, no se ha encontrado aún un modo mejor de generar conocimiento, sino mediante la investigación científica, por esta razón, en la sociedad actual las instituciones del conocimiento, en particular las universidades, han adquirido una importancia estratégica donde la distribución social de los beneficios de la ciencia y la tecnología siguen un patrón similar al de la distribución de la riqueza y el poder en el mundo. Consiguientemente, las sociedades capaces de movilizar sus instituciones educativas, de investigar y gestionar los flujos de conocimiento, enfocan sus esfuerzos en los fundamentos metodológicos del Aprendizaje. 
Dentro del aprendizaje hay una tipología y metodología denominada "significativa", la cual se puede definir como aquella que permite construir su propio aprendizaje y, además, la dota de significado. Dicho de otra manera, el aprendizaje significativo no se olvida y se mantiene en las capacidades del alumno. Eso sí, los alumnos son los responsables de su propio aprendizaje, con lo que juegan un papel activo y participativo. Con todo, son muchas las ventajas de introducir esta metodología en la Educación Superior y los programas de postgrado en particular, pues el aprendizaje significativo, permite que el estudiante construya su propio aprendizaje, lo que en perspectiva motiva a la adquisición de una cultura investigativa y la consiguiente producción científica.

La universidad cada vez más se afianza como fuente de producción científica, cuya tendencia a nivel global se orienta en ampliar su función investigativa, no solo para educar a los estudiantes, sino también para el desarrollo profesional de los docentes en investigación y proveerles de herramientas conceptuales, procedimentales y aptitudes necesarias para analizar su entorno y construir conocimiento mediante la práctica de metodologías orientadas.

Consiguientemente, ello obliga a que sea necesario adentrarse en la teoría en sí y profundizar en la misma, de manera que se aprenda significativamente para, con ello, lograr que los aprendizajes a desarrollar en los alumnos, relativos a los contenidos científica y contextualmente validados, sean realmente significativos. Básicamente el análisis está referido a utilizar los conocimientos previos del alumno para construir un nuevo aprendizaje, en donde el docente de los programas de postgrado se convierte sólo en el mediador entre los conocimientos y los alumnos, participando en lo que aprenden; pero para lograr la colaboración del alumno se deben crear estrategias que permitan que el alumno se halle dispuesto y motivado para aprender.

Una de las grandes asignaturas pendientes y urgentes de las universidades es el desarrollo de la Cultura investigativa en los programas de postgrado.

Se debe entender por tanto qué, la calidad de la educación universitaria está íntimamente asociada con el proceso de aprendizaje y la práctica de la investigación, teniendo como finalidad fomentar la cultura investigativa como también la producción científica donde los docentes y estudiantes se agrupan propiciando intercambios de saberes mediante proyectos de investigación. En otras palabras, los protagonistas son los que deben hacer investigación, consumir investigación y utilizarla pertinentemente 
en el desarrollo de los programas de postgrado.

Las universidades latinoamericanas en su mayoría carecen de genuinos procesos de transferencia de conocimiento para formar investigadores, vale decir que puedan identificarse con los temas y especialidades de interés, para dar validez y confiabilidad a las investigaciones realizadas. Los problemas en el mayor de los casos están asociados a la falta de Aprendizajes Significativos en las fases iniciales de los procesos de adquisición del conocimiento.

En síntesis, es evidente que los programas de postgrado de las universidades tienen urgente necesidad de una verdadera cultura investigativa para docentes y estudiantes, tomando en cuenta que la producción científica es fundamental para el desarrollo de la ciencia y la tecnología del país.

\section{EL APRENDIZAJE Y LA INVESTIGACION EN LA EDUCACION SUPERIOR}

Estamos en la era de la información donde la educación superior y la investigación científica representan el rumbo a seguir para el desarrollo y progreso mundial. Las universidades contextualizadas en espacios sociales con características temporales definidas por circunstancias culturales que les imprimen un carácter distintivo, se convierten singularmente en una diversidad dentro de la generalidad macro-social que las contiene.

Así, los nuevos paradigmas que envuelven la sociedad global, redefinen el actuar de las instituciones que a ella le conciernen. Por ello, los estudiosos del desarrollo de la actividad científica de las educación superior se han visto impelidos a orientar sus investigaciones hacia el criterio de la pertinencia de su accionar frente a los cambios impuestos por el desarrollo científico, tecnológico y de innovación sin precedentes, de cuyos alcances las universidades han sido protagonistas relevantes, para examinar su rol en las transformaciones epistemológicas, tecnológicas y pedagógicas en la sociedad global, en el contexto de la transdisciplinariedad y diversidad.

El nuevo paradigma impone a estas organizaciones, desarrollar planes, programas y estructuras curriculares para que los estudiantes desarrollen capacidades científicas para aprender a aprender, construir y resolver los problemas de la diversidad del entorno social y natural. De allí, que ellas no pueden circunscribirse solo a enseñar a usar las nuevas herramientas tecnológicas, sino involucrarse en un programa de desarrollo del conocimiento científico, de la importancia tecnológica y los principios que en ellas se 
involucran. Uno de los pilares principales de esta temática radica precisamente en el aprendizaje y los principios metodológicos con los que se imparte en las Universidades.

\section{EL APRENDIZAJE SIGNIFICATIVO Y EL CONOCIMIENTO EN LOS PROCESOS INVESTIGATIVOS}

El aprendizaje humano va más allá de un simple cambio de conducta, conduce a un cambio en el significado de la experiencia. Durante mucho tiempo se consideró que el aprendizaje era sinónimo de cambio de conducta, esto, porque dominó una perspectiva conductista de la labor educativa; sin embargo, se puede afirmar con certeza que el aprendizaje humano va más allá de un simple cambio de conducta, conduce a un cambio en el significado de la experiencia.

Actualmente las palabras al uso son aprendizaje significativo, cambio conceptual y constructivismo. Una buena enseñanza debe ser constructivista, promover el cambio conceptual y facilitar el aprendizaje significativo.

Hablar de "Aprendizaje Significativo" equivale, ante todo, a poner de relieve el proceso de construcción de significados como elemento central del proceso de enseñanza/aprendizaje. De acuerdo a Moreira (2014), no hay enseñanza sin captación de significados, en otras palabras, no hay enseñanza sin aprendizaje. Notables investigadores del Aprendizaje significativo como: Ausubel (1976); Ausubel (2002); Bustos y Otros (2010); Castillo y Otros (2006); Diaz y Otros (2004), Ojeda (2007), Ojeda y Otros (2012), Palacios y Otros (2004) Pimienta (2008), sostienen que existe un cambio notable en la enseñanza, colocando como el centro de la educación al estudiante y su esfuerzo por aprender significativamente.

Por tanto, el valor del aprendizaje significativo reside en la posibilidad que ofrece el docente para indagar en la realidad en busca de formas más profundas de comprensión, y no tanto en su capacidad para prescribir cursos de acción.

El concepto del Aprendizaje Significativo, por tanto, puede resumirse en las siguientes dos tesis:

- En primer lugar, el concepto de aprendizaje significativo, tal como aparece en las formulaciones de Ausubel, implica un cambio de perspectiva en la solución dada al clásico problema pedagógico de la preparación o disponibilidad para el aprendizaje en la Educación Superior. 
- En segundo lugar, el concepto de aprendizaje significativo supone, ante todo, un cambio de perspectiva radical en la manera de entender el proceso de enseñanza/aprendizaje. Frente a la concepción tradicional y habitual de que el aprendizaje del alumno depende directamente de la influencia del docente y de la metodología de enseñanza utilizada, se pone de relieve la importancia del conocimiento previo del alumno y, en general, de sus procesos de pensamiento.

La realidad de la Universidad pública de los países de América Latina y en particular de Bolivia, nos muestra que los modelos predominantes en la enseñanza de los programas de postgrado tienen un carácter tecnicista, ya que enfatizan el desarrollo de competencias orientadas en la resolución instrumental de los problemas de la práctica profesional.

Analizando los procesos de formación, hay factores que afectan a la educación que se imparte en los programas de postgrado impactando de manera determinante en la satisfacción de las necesidades básicas de aprendizaje que requiere el país, entre las que se puede señalar prácticas pedagógicas inadecuadas y estáticas frente a las exigencias de cambio, aquellas que se encuentran ligadas a la pedagogía tradicional, en la que el aprendizaje es memorístico, por lo tanto de corta duración, es impuesta al alumnado, en cuya práctica docente los alumnos copian pasivamente los dictados, memorizan sin pensar ni entender, no son participativos y solo responden cuando el docente les pregunta, trabajan solos sin cooperarse entre sí, no participan por iniciativa propia, no arriban a conclusiones, no investigan, no exponen y discuten sus ideas, no descubren significados, no proponen alternativas y no resuelven problemas (Alvarado Resendiz, y otros).

Los docentes y estudiantes de los programas de postgrado se enfrentan al reto de integrar en la enseñanza y el aprendizaje de las disciplinas habilidades y competencias que van desde la resolución de problemas prácticos relacionados con la profesión, hasta el dominio de habilidades cognitivas y lingüísticas relacionadas con las estructuras de sus propias áreas profesionales, por esta la razón, surge el análisis del papel que desempeña el docente y la metodología con el uso del aprendizaje significativo.

Al dar una mirada a lo que ha sido la educación superior se puede ver cómo los procesos de formación especialmente en los programas de postgrado, se han 
caracterizados por estar centrados en la enseñanza más que en el aprendizaje. En ese sentido, la misión de la educación superior no es tanto enseñar al alumno una multitud de conocimientos que pertenecen a campos muy especializados, sino, ante todo, aprender a aprender, procurar que el alumno llegue a adquirir una autonomía intelectual. Dar pie a estimular el aprendizaje en el aula, entendiendo por éste, un aprendizaje significativo que abrirá los horizontes investigativos para la producción científica.

Consiguientemente, una formación crítico-reflexiva, propia del contexto de la Educación Superior, requiere necesariamente de iniciativas para fomentar la curiosidad y conseguir que tanto los estudiantes como profesores sean capaces de cuestionar las situaciones que los rodean recurriendo a la creatividad y a la innovación y valorando la incertidumbre como un aspecto potencial de la realidad.

Una de las estrategias para encarar las deficiencias del Aprendizaje Significativo exige un nuevo perfil docente que pueda satisfacer las demandas que estos cambios producen, donde la capacidad de reflexionar sobre la propia práctica y la habilidad para desarrollar el pensamiento reflexivo se consideran aspectos clave. Concretamente, en este momento se pide un nuevo perfil docente que pueda fomentar en el alumnado aprendizajes significativos, habilidades de pensamiento superior, el aprender a aprender, mediante la revisión del ejercicio profesional y la habilidad del pensamiento reflexivo, capaces de constituirse metodológicamente en la base de la producción científica.

\section{EL ROL INVESTIGATIVO DE LOS POSTGRADOS EN LAS UNIVERSIDADES BOLIVIANAS}

El ecosistema investigativo en Bolivia principalmente está determinado por el entorno institucional de la investigación científica a cargo del Sistema Estatal de Ciencia y Tecnología del Ministerio de educación que tiene como objetivo alcanzar "la Soberanía Científico y Tecnológica con Identidad Propia” (Plan nacional de ciencia tecnología e innovación. Ministerio de educación, Bolivia, 2013). Para lograr este fin, se han programado algunas acciones prioritarias para permitir el "Acceso a información Científica y Tecnológica gratuita para los investigadores”.

De acuerdo a los informes oficiales, desde 2008, se cuenta con un financiamiento gubernamental que permite a los Centros e Institutos de Investigación, a nivel nacional, 
asegurar el acceso a la información científica internacional a todos los grupos de investigación del país, en forma equitativa, a través de 13 casas editoriales en las que se ofertan más de 200.000 revistas científicas de alcance mundial, en todas las áreas del conocimiento y al que tienen acceso gratuito todas las universidades bolivianas (desde el inicio las descargas han alcanzado un número de 80.000 artículos científicos).

El sistema permite la difusión y divulgación de la información científica y tecnológica generada en Bolivia, a través de revistas científicas nacionales y su vinculación con el resto del mundo. Estas iniciativas, de acuerdo a las autoridades de gobierno, deberían "promover el acceso e intercambio de información científica y tecnológica producida localmente y generada por mecanismos y estrategias para su comunicación a un público mayor. Asimismo, se informa que existe un trabajo periódico en la publicación del potencial científico tecnológico que muestra los recursos humanos, la infraestructura y los trabajos de investigación con los que cuentan los centros e institutos de investigación en el país".

Sin embargo, la realidad de los datos de producción científica que se ofrecen más adelante muestra una realidad totalmente distinta a las buenas intenciones de los organismos gubernamentales.

Otro de los elementos del ecosistema investigativo corresponde a la normativa de la investigación científica vigente en la universidad boliviana.

La Universidad Boliviana en su XI CONGRESO NACIONAL DE UNIVERSIDADES (Oruro-Bolivia), aprobó el MODELO ACADEMICO DEL SISTEMA DE LA UNIVERSIDAD BOLIVIANA (CEUB, 2011), en la que ratifica su compromiso de integración con la sociedad, constituyéndose en el motor del desarrollo productivo, económico y social, basado en sólidos cimientos de la investigación científica, el desarrollo tecnológico y la innovación. Todo ello con la finalidad de contribuir a la mejora de las condiciones de vida, calidad del desarrollo humano y el desarrollo sostenible, formulando un modelo académico viable, sostenible y medible en función a las características de la realidad nacional, regional y local.

Aparte de estos dos instrumentos que norman y promueven en desarrollo de la investigación científica en Bolivia, existen normas propias de las Universidades Públicas que ratifican el carácter prioritario de la investigación científica, sin embargo la realidad que expresan informes investigativos de distintas organizaciones dedicadas 
al monitoreo de la Educación Superior y las entidades gubernamentales de investigación nos muestra una realidad no muy alentadora, como veremos en la descripción del estado de la investigación científica en Bolivia.

\section{La educación postgradual en el desarrollo de la investigación científica}

La calidad y la factibilidad de la investigación científica y el desarrollo tecnológico de un país dependen fundamentalmente de la existencia de recursos humanos de alta formación académica (nivel de $\mathrm{PhD}$ ). Entonces, la educación postgradual aparece como una cuestión esencial para el desarrollo de Bolivia.

A partir de los datos analizados sobre los procesos investigativos en la Educación superior en Bolivia, queda claro que los programas de postgrado no están diseñados para promover la investigación científica, por tanto, no permiten una actividad académica suficiente.

Una de las distorsiones principales de los programas de postgrado en Bolivia tiene que ver en la falta cultura investigativa y las deficiencias metodológicas de los procesos de aprendizaje. Este problema de "principio" influye de manera determinante en la vocación investigativa de los programas de postgrado y su escasa producción científica.

A pesar de que existe una marcada diferencia entre el tipo de aprendizaje orientado a la investigación en los diferentes programas de posgrado en el ámbito internacional, en los programas de postgrado de Bolivia esta diferencia no se da. Esto lleva a que se introduzca el componente de investigación en los planes de estudio como asignaturas o talleres que buscan fortalecer las competencias de los estudiantes en el área de investigación, sin embargo, al no tener claridad sobre lo que se debe saber y lograr con la investigación en cada postgrado, los espacios pedagógicos están determinados antojadizamente.

Las asignaturas de formación profesional ocupan más del $90 \%$ de las asignaturas que se cursan en un programa de postgrado (eso incluye también a los doctorados), y que las asignaturas de investigación se imparten de manera tradicional a través de módulos, talleres y seminarios. Esto es una muestra más del énfasis (orientación) de los postgrados donde se aprecia la inclinación hacia la profesionalización y no hacia la investigación. La investigación en todo caso se enseña como una asignatura más y no como una práctica académica, es decir, los estudiantes no están en los laboratorios o 
haciendo pasantías, o realizando investigaciones, o participando en congresos de investigación o escribiendo y discutiendo artículos científicos.

A pesar de la diversificación de los programas de postgrado en el sistema universitario de Bolivia el mayor desarrollo de producción científica se desarrolla fuera de los mismos. Esta divergencia se debe a que por lo general los cursos de postgrado no se hallan directamente vinculados a los centros de investigación; sin embargo, al ser las maestrías y doctorados mayormente concentrados en el área social y de ciencias económicas, que requieren de menor infraestructura y equipamiento; son las que han cobrado mayor peso en este campo. Muchas de estos programas de postgrado tienen un carácter más bien profesionalizante que de investigación, por lo que su articulación con las estructuras de investigación sigue siendo débil.

Consiguientemente, los resultados de producción científica evidencian un número muy bajo de publicaciones por parte de los programas de postgrado en Bolivia. Una de las causas frecuentes tiene que ver con que los estudios de postgrados se orientan en temáticas referentes a la caracterización de sus problemáticas, perfilar los potenciales estudiantes, políticas nacionales de incremento de doctores o magíster y en temas de autoevaluación y acreditaciones.

El bajo número de publicaciones también se deduce en que la experiencia de las universidades no incentiva a los docentes a la investigación y por otra parte los programas de postgrado no exigen publicaciones científicas como requisitos de admisión y graduación.

Mientras la articulación de la formación en postgrados con la investigación de alto nivel y de la universidad con el sector empresarial y la sociedad puede ser considerada como una tendencia emergente en la mayoría de los países de América Latina, y ya está consolidada en las universidades referentes en el ámbito mundial, en Bolivia está ausente en los programas de postgrado y en la práctica de producción científica curricular.

Mientras las nuevas vertientes de los doctorados y maestrías surgen de la demanda del mercado que espera procesos de formación más cortos, centrados en temáticas más específicas y que permitan al estudiante no solo concentrarse en desarrollar capacidades en investigación, sino en un entrenamiento para lograr mejores desempeños laborales; los programas de postgrado en Bolivia no se orientan en el 
desarrollo de capacidades investigativas de los estudiantes y priorizan el sistema tradicional de distribuir la carga formativa de aula.

La combinación de toda la serie de factores descritos anteriormente, afecta el nivel de producción científica y la generación de conocimientos en las universidades de Bolivia. Contrario a lo que muchos piensan, en Bolivia la baja producción científica no se debe a la falta de talento, que sí existe, sino a la falta de posibilidades para la explotación del mismo. Las investigaciones requieren financiamiento; si no lo hay, es poco probable que puedan llevarse a cabo de manera adecuada. Si no surgen proyectos, entonces la oferta laboral científica se reduce, y si hay poco trabajo, entonces muchos cerebros deberán escoger entre otros sectores de su interés para trabajar, o salir de su país para poder ejercer.

El desafío de la educación superior en Bolivia y de los programas de postgrado en particular, es contribuir al avance del conocimiento científico, que se traduzca en propuestas tecnológicas destinadas a colaborar en la resolución de los problemas que afectan a nuestra sociedad. La universidad boliviana debe estar a la altura de sus responsabilidades, no existe otra alternativa si quiere contribuir al desarrollo. En suma, a las universidades bolivianas les queda todavía mucho camino por recorrer.

\section{CONCLUSIONES}

La Universidad Boliviana es esencialmente profesionalizante, es explicable por tanto, que los estudios de pregrado siendo en lo fundamental profesionalizantes no pongan énfasis en la investigación y, por ende, en la producción científica. Empero, lo inadmisible resulta del hecho de que los programas de postgrado sean, también, profesionalizantes. Desde esa óptica, la Universidad boliviana, no forma futuros líderes, ni pensadores, ni investigadores, sino "profesionales" que ejerzan, no que piensen.

La formación de investigadores por parte de los programas de postgrado constituye una falencia. Los encargados de dirigir esta enseñanza carecen, en la mayoría de los casos, de la experiencia para hacerlo. Los programas de postgrado en Bolivia conservarán sus debilidades estructurales mientras los titulares de la docencia no incursionen ellos mismos en la construcción de conocimiento que derive en la producción de ciencia, tecnología e innovación.

No queda duda que, en los programas formativos de los postgrados en Bolivia, los 
problemas de aprendizaje significativo influyen de manera determinante en los problemas de la escasa investigación, el predominio del aprendizaje a corto plazo en las aulas de pre y postgrado son determinantes a la hora de identificar los problemas que impactan en la escasa investigación científica.

Consiguientemente, comprender el pensamiento de los alumnos de los programas de postgrado podría dar una mayor capacidad de adaptar los métodos de enseñanza a sus aptitudes, comprendiendo que, en los estudiantes de postgrado, varían en gran medida tanto su nivel de desarrollo cognoscitivo como su conocimiento académico, situación que demuestra que los alumnos son la mejor fuente de información acerca de sus propias habilidades y competencias.

Las instituciones de educación superior y los programas de postgrado en particular, deben estar comprometidos tanto en la incorporación de métodos de Aprendizaje Significativo, como en la capacitación de sus docentes, para que su esfuerzo se vea enfocado en lograr que los estudiantes sean aprendices eficaces, autónomos y sobre todo practiquen de la mejor manera la autogestión.

De acuerdo con las teorías expuestas en este trabajo, el conocimiento es construido a partir de las experiencias individuales, de ahí la importancia de analizar las ideas y percepciones que el docente universitario tiene en relación a las estrategias de enseñanza respecto al aprendizaje significativo, lo que de manera consecuente puede tener un impacto positivo en el desarrollo de una cultura investigativa apropiada para aportar en la producción científica.

La creación de conocimiento no garantiza por sí misma un crecimiento económico y una mejora en el bienestar social, siendo necesaria, la difusión de ese conocimiento y su aprovechamiento por la comunidad científica e industrial. La relevancia social de la investigación, se justifica por la necesidad de generar mejoras en la calidad de vida, bienestar social y desarrollo del Estado Boliviano, a partir de la aplicación de herramientas de gestión estratégica, administrativa, operativa y comercial, que coadyuven a la consolidación de Centros de Investigación Universitarios competitivos, prósperos y sostenibles. 


\section{BIBLIOGRAFÍA}

Aguerrondo Inés (2017). El Nuevo Paradigma de la Educación para el siglo XXI.

Fuente: OEI. Programas. Desarrollo Escolar y Administración Educativa. Buenos

Aires, 12 de junio de 1999- http://www.oei.es/administracion/aguerrondo.htm

Aguirre Arias Erika Lizeth. UNA MIRADA DESDE EL APRENDIZAJE

SIGNIFICATIVO EN COMPARACIÓN A UN MODELO PEDAGÓGICO TRADICIONAL, PARA LA ENSEÑANZA DEL CONCEPTO BIOLÓGICO DE POBLACIÓN Y ANALISIS DE LOS PROBLEMAS RELACIONADOS AL PROCESO ENSEÑANZAAPRENDIZAJE A ESTUDIANTES DE NOVENO

GRADO. Universidad Distrital Francisco José de Caldas, Bogotá D.C. https://www.saece.com.ar/docs/congreso5/trab003.pdf

Alvarado Resendiz José Luis, García Munguía Mónica, Castellanos López Liliana Yadira. “Aprendizaje Significativo En La Docencia De La Educación Superior”, Uiversidad Autónoma del estado de Hidalgo, MEXICO. https://www.uaeh.edu.mx/scige/boletin/tlahuelilpan/n9/e1.html

Ausubel D. (1983). Teoría Del Aprendizaje Significativo. Recuperado el 05 de julio de 2016:

http://delegacion233.bligoo.com.mx/media/users/20/1002571/files/240726/Aprendi zaje_significativo.pdf

Ausubel, D. P. (2002). Adquisicion y retencion del conocimiento. Una perspectiva cognitiva. Barcelona, España: Ediciones Paidos Iberoamerica, S.A.

Ausubel, D. (1976). Psicología educativa: un punto de vista cognoscitivo. México: Editorial Trillas.

Ausubel David P. (2002). Adquisición y retención del conocimiento: una perspectiva cognitiva/ traducción de Genis Sánchez Barberán. Barcelona, España: Ediciones Paidós Ibérica, 200. ISBN: 8449312345

Barriga Arceo F. D., Hernández Rojas G. (2010). Estrategias Docentes Para Un Aprendizaje Significativo: Una Interpretación Constructivista. Mc Graw Hill. México D.F.

Bustos, A., Coll, C., Córdoba, F., Rey, R. Del Engel, A., Escaño, J., Rochera, M. J. (2010). Desarrollo, aprendizaje y enseñanza en la educación secundaria, Barcelona: Editorial Graó. 
Castillo, C. V., Yahuita, J., Garabito, R., (2006). Estrategias docentes para un aprendizaje significativo. Cuad. - Hosp. Clín., La Paz, 51, (1), 96-101. Recuperado de

<http://www.revistasbolivianas.org.bo/scielo.php?script=sci_arttext\&pid=S1652$67762006000100015 \& \operatorname{lng}=\mathrm{es} \& n r m=$ iso $>$ [ Links ]

Diaz, F., y Hernandez, G. (2004). Estrategias docentes para un aprendizaje significativo. Mexico: Editorial McGraw Hil

Educación superior en Bolivia Elaboración (2007). Temas de debate PIEB- NÚMERO 7, AÑO 4 FEBRERO DE 2007. www.pieb.org | d.1.: 4-2-794-03. La Paz-Bolivia

Jiménez Landívar Elizabeth. Investigación en las universidades bolivianas: una asignatura pendiente. domingo, 08 de abril de 2018, https://www.paginasiete.bo/ideas/2018/4/8/investigacion-en-las-universidadesbolivianas-una-asignatura-pendiente-175622.html

Montalvo, E. (2006). Fundamentos Psicológicos del Proceso de Enseñanza.

Aprendizaje. Extraído el 13 de noviembre de 2006 de: http://www.ueb.edu.ec

Ojeda, A., Díaz, F. E., González, L., Pinedo, P., y Hernández M. (2007). Los mapas conceptuales: una poderosa herramienta para el aprendizaje significativo. ACIMED, $15(5)$ Recuperado de http://scielo.sld.cu/scielo.php?script=sci_arttext\&pid=S1024$94352007000500009 \& \operatorname{lng}=$ es\&tlng=es.

Ojeda, A., Díaz, F.E., González, L., Pinedo, P. H., (2012). Los mapas conceptuales: una poderosa herramienta para el aprendizaje significativo, 15(5). Recuperado de http://bvs.sld.cu/revistas/aci/vol15_5_07/aci09507.htm

Palacios, J., Marchesi, Á., y Coll, C. (2004). Desarrollo psicológico y educación: Psicología evolutiva. Barcelona: Editorial Alianza

Pimienta, J. H. (2008b). Constructivismo. Estrategias para aprender a aprender. México: Editorial Pearson Educación de México S.A. de C.V.

Plan nacional de ciencia tecnología e innovación (2013). Ministerio de educación, Bolivia.https://www.minedu.gob.bo/files/publicaciones/vcyt/dgcyt/PLAN-

NACIONAL-CyT.pdf

XI CONGRESO NACIONAL DE UNIVERSIDADES (2011). MODELO ACADEMICO DEL SISTEMA DE LA UNIVERSIDAD BOLIVIANA 
2015-2019,

CEUB.http://www.planificacion.umsa.bo/documents/1778193302/0/CEUB+MOD ELO+ACADEMICO+SUB.pdf

Sanfeliciano Alejandro (2019).Aprendizaje significativo: definición y características.

31 mayo, 2019. https://lamenteesmaravillosa.com/aprendizaje-significativo-definicioncaracteristicas/

Vigotsky Lev, Piaget Jean (2012). TEORÍAS DEL APRENDIZAJE. IFD Tacuarembó, Mayo/2012. https://profesorailianartiles.files.wordpress.com/2013/04/piaget-y-vigotsky.pdf 